\title{
Origen y Trayecto Anómalos del Nervio Musculocutáneo
}

\author{
Anomalus Origin and Traject of Musculocutaneous Nerve
}

Jorge Roberto Marcante Carlotto; Luciana Estacia Ambros; Juarez Antônio Dal Vesco \& Paulo Roberto Reichert

CARLOTTO, J. R. M.; AMbROS, L. E.; DAL VESCO J. A. \& REICHERT, P. R. Origen y trayecto anómalos del nervio musculocutáneo. Int. J. Morphol., 27(2):507-508, 2009.

RESUMEN: El nervio musculocutáneo se origina del fascículo lateral en el plexo braquial y sus fibras emergen principalmente de las raíces nerviosas anteriores de C5 y C6. Durante la disección de rutina del plexo braquial en un cadáver formolizado, fue encontrada una variación del origen y trayecto de este nervio. El nervio musculocutáneo se originaba de la cara lateral del nervio mediano y después del origen, cruzaba anteriormente al músculo coracobraquial, sin perforarlo, de medial hacia lateral y de proximal hacia distal, para después ramificarse. Las variaciones anatómicas encontradas contribuyen para el estudio de la anatomía y sirven para el cirujano en intervenciones en la fosa axilar y en la parte anterior del brazo, previniendo, así, complicaciones operatorias.

PALABRAS CLAVE: Anatomía; Plexo Braquial; Nervio Musculocutáneo.

\section{INTRODUCCIÓN}

El nervio musculocutáneo se origina del fascículo lateral del plexo braquial junto con el ramo lateral del nervio mediano. Sus fibras derivan principalmente de C5 y C6 con aporte de C7 (Moore \& Dalley, 2001). Es responsable por la inervación de los músculos coracobraquial, bíceps braquial y braquial y por la inervación sensitiva de las caras anterior y posterolateral del antebrazo (Gray \& Goss, 1988).

Han sido descritas variaciones cuanto al origen y trayecto del nervio musculocutáneo, a pesar que la mayoría presenta su origen directo del fascículo lateral del plexo braquial. El nervio musculocutáneo puede tener su origen de los fascículos lateral y posterior, del nervio mediano u originarse solamente del fascículo posterior (Latarjet \& Liard, 1996; Tountas \& Bergman, 1993). El nervio pasa a través de las fibras del músculo coracobraquial, de posterior para anterior y es conocido como Nervio Perforante de Caserio (Testut \& Latarjet, 1968), sin embargo la no transfijación de este nervio al músculo coracobraquial ya ha sido relatada.

\section{RELATO DE CASO Y DISCUSIÓN}

Durante la disección de rutina del plexo braquial izquierdo de un cadáver de un individuo adulto, de sexo masculino, de edad media, fijado en formalina, perteneciente al Departamento de Anatomía Humana de la Facultad de Medicina de la Universidad de Passo Fundo, fue encontrada una variación anatómica del origen y trayecto del nervio musculocutáneo. Este nervio se originaba de la cara lateral del nervio mediano, el cual presentaba su formación patrón, a nivel de transición del tercio proximal con el medio del brazo (Fig. 1). El nervio musculocutáneo cruzaba anteriormente al músculo coracobranquial, de medial a lateral y de proximal a distal, sin perforarlo, para terminar como nervio cutáneo lateral del antebrazo. Los otros nervios originados de ambos plexos braquiales izquierdo y derecho no presentaban variaciones anatómicas.

Variaciones del plexo braquial son relatadas con frecuencia en la literatura, pero el origen del nervio musculocutáneo a partir del nervio mediano raramente es encontrado. Ese origen anómalo tiene una explicación embriológica, una vez que el plexo braquial aparece como un cono radicular único del miembro superior, que se divide longitudinalmente en segmentos ventral y dorsal. El segmento ventral será el responsable por dar origen a los nervios mediano y musculocutáneo, ya que ambos provienen del mismo segmento estando sujetos a variaciones durante su proceso de división embriológicos (Iwamoto et al., 1990).

De acuerdo con el estudio de Tountas \& Bergaman, sobre el origen del nervio musculocutáneo, quienes analizados 75 casos, solamente $2 \%$ de estos presentaban origen a partir de nervio mediano, representando así la segunda variación más rara, ya que del fascículo posterior del plexo braquial se origina en el $1,4 \%$. 
En relación al trayecto del nervio musculocutáneo éste puede pasar posterior al músculo o entre él y la cabeza corta del músculo bíceps braquial y, de vez en cuando, puede también atravezar al músculo braquial y a la cabeza corta del músculo bíceps. Tountans \& Bergman y Testut \& Latarjet disecaron 100 plexos braquiales y señalaron la no transfijación del músculo coracobraquial por el nervio musculocutáneo en el 6,5\% y 3,4\% de los casos estudiados, respectivamente. Sin embargo, puede ocurrir la división del músculo coracobraquial por el nervio musculocutáneo en porciones absolutamente distintas, formando el ligamento braquial medial. Este ligamento se origina del tubérculo mayor del número y se prolonga hasta la extremidad distal del músculo coracobraquial (Testut \& Latarjet).

Con respecto a otras variaciones frecuentes del nervio musculocutáneo, éste puede estar ausente (Song et al., 2005), estar duplicado o presentar anastomosis con el nervio mediano (Olave et al. (2000).

Conocer las variaciones del plexo braquial sirve de alerta y orientación para evitar lesiones nerviosas durante los procedimientos diagnósticos y terapéuticos realizados en esta región.

CARLOTTO, J. R. M.; AMBROS, L. E.; DAL VESCO J. A. \& REICHERT, P. R. Anomalus origin and passage of musculocutaneous nerve. Int. J. Morphol., 27(2):523-524, 2009.

SUMMARY: The musculocutaneous is originated from the lateral fascicle in the brachial plexus and its fibers emerge mainly from the anterior nervous roots C5 and C6. During the routine dissection of the brachial plexus in a formolized corpse, its origin variation and passage was found. The nerve was originated on the lateral face of the median nerve and after its origin, crossed anteriorly to the coracobrachial muscle, without perforating it, from medial to lateral, proximal to distal, after to branch off. The anatomical variations found contribute to the anatomy study and they serve as alert for the surgeon in interventions in the axillary's cavity and in the previous store of the arm, preventing, thus, operational complications.

KEY WORDS: Anatomy; Brachial Plexus; Musculocutaneous nerve.

\section{REFERENCIAS BIBLIOGRÁFICAS}

Gray, H. \& Goss, C. M. Anatomia. 29ª ed. Rio de Janeiro, Guanabara Koogan, 1988.

Iwamoto, S.; Kimura, K.; Takahashi, Y. \& Konishi, M. Some aspects of the communicating branch between the musculocutaneous and median nerves in man. Okajimas Folia Anat. Jpn., 67(1):47-52, 1990.

Latarjet, M. \& Liard, A. R. Anatomia Humana. $2^{\text {a }}$ ed. São Paulo, Panamericana, 1996.

Moore, K. L. \& Dalley, A. F. Anatomia: orientada para clínica. $4^{\mathrm{a}}$ ed. Rio de Janeiro, Guanabara Koogan, 2001.

Olave, E.; Gabrielli, C.; Braga, M. T. T.; Del Sol, M. \& De Souza, A. Ramo comunicante entre los nervios musculocutáneo y mediano en el Hombre. Rev. Chil. Anat., 18(2):301-4, 2000.

Testut, L. \& Latarjet, A. Tratado de anatomía humana. 9a ed. Barcelona, Salvat, 1968.

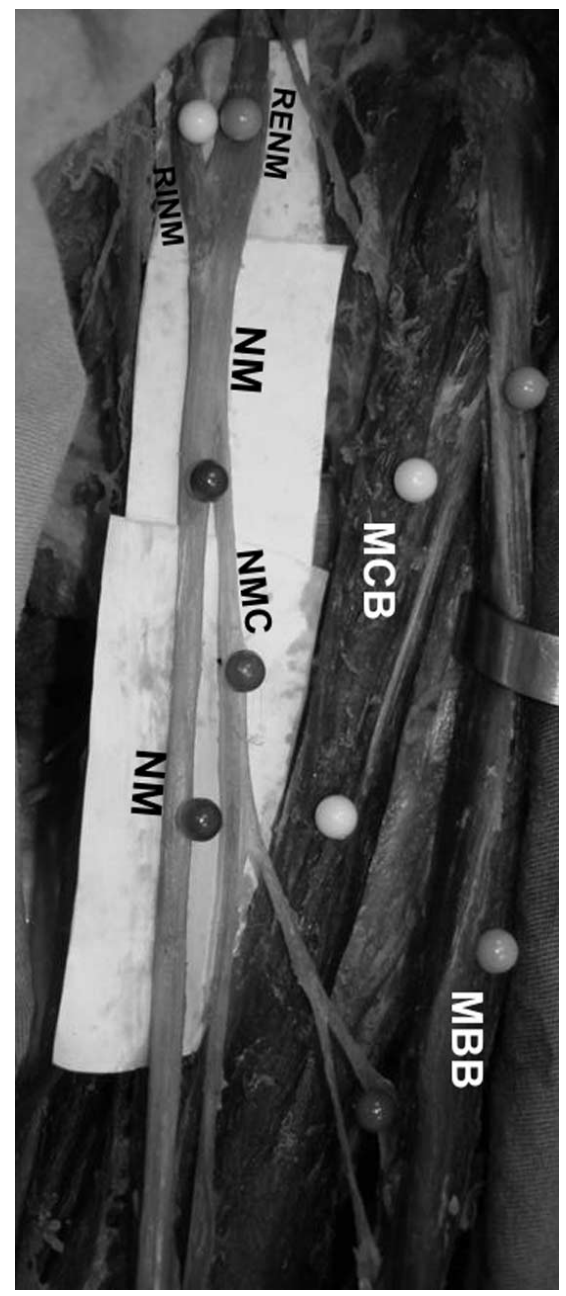

Fig. 1. Fotografía de la disección del plexo braquial izquierdo. RENM: Raíz lateral del nervio mediano. RINM: Raíz medial del nervio mediano. NM: Nervio mediano. NMC: Nervio musculocutáneo. MCB: Músculo coracobraquial. MBB: Músculo bíceps braquial.

Tountas, C. \& Bergman, R. Anatomic variations of the upper extremity. New York, Churchill Livingstone, 1993.

Song, W. C.; Jung, H. S.; Kim, H. J.; Shin, C.; Lee, B. Y. \& Koh, K. S. A Variation of the Musculocutaneous Nerve Absent. Yonsei Med. J., 44(6):1110-3, 2003.

Dirección para correspondencia: Jorge Roberto Marcante Carlotto Rua Fagundes dos Reis, 466 - Edifício Toulose Lautrec Apartamento 403, Bairro Centro CEP: $99010-070$

Passo Fundo - Rio Grande do Sul BRASIL

E-mail: jorgecarlotto@hotmail.com Recibido : 05-09-2008 Aceptado: 24-02-2009 